\title{
Laboreal
}

Volume $7 \mathrm{~N}^{\circ} 1$ | 2011

Psicodinâmica e psicopatologia do trabalho

\section{Texto Introductorio : Psicopatología del trabajo - Psicodinámica del Trabajo}

Texto Introdutório : Psicopatología do trabalho - Psicodinámica do Trabalho

Texte d'Introduction: Psychopathologie du travail - Psychodynamique du

travail

Introductory Text : Psychopathology of work - Psychodynamics of work

\section{Christophe Dejours}

\section{OpenEdition}

\section{Journals}

Edición electrónica

URL: http://journals.openedition.org/laboreal/8030

DOI: $10.4000 /$ laboreal. 8030

ISSN: 1646-5237

\section{Editor}

Universidade do Porto

\section{Referencia electrónica}

Christophe Dejours, « Texto Introductorio : Psicopatología del trabajo - Psicodinámica del Trabajo », Laboreal [En línea], Volume $7 N^{0} 1$ | 2011, Publicado el 01 julio 2011, consultado el 23 septiembre 2020. URL : http://journals.openedition.org/laboreal/8030 ; DOI : https://doi.org/10.4000/laboreal.8030

Este documento fue generado automáticamente el 23 septiembre 2020.

\section{cc) (i) (8)}

Laboreal está licenciado com uma Licença Creative Commons - Atribuição-NãoComercial 4.0 Internacional. 


\title{
Texto Introductorio : Psicopatología del trabajo - Psicodinámica del Trabajo
}

\author{
Texto Introdutório : Psicopatología do trabalho - Psicodinámica do Trabalho \\ Texte d'Introduction: Psychopathologie du travail - Psychodynamique du \\ travail \\ Introductory Text : Psychopathology of work - Psychodynamics of work
}

Christophe Dejours

1 Aunque los primeros problemas vinculados a la relación entre el trabajo y la salud mental en Francia hayan surgido entre dos grandes guerras, fue sólo a partir del final de la Segunda Guerra Mundial que la investigación clínica en psicopatología del trabajo verdaderamente comenzó (Billiard, 2011). En un primer momento, estos estudios pretendían identificar las patologías mentales específicas de determinados tipos de trabajo (como las neurosis de las telefonistas descritas por J. Bégoin en 1957), pero resultó necesario admitir que no existía ningún síndrome psicopatológico exclusivamente producido por las dificultades del trabajo, contrariamente a lo que puede acontecer en el caso de algunas enfermedades físicas, causadas exclusivamente por la contaminación del entorno laboral (como el saturnismo relacionado con los vapores de plomo, la silicosis de los mineros de carbón, ...)

2 Se hizo posible extender la investigación clínica después que se identificó el conflicto específico entre los problemas derivados de la organización del trabajo y el funcionamiento psicológico de los trabajadores. Así, algunas formas de organización del trabajo surgieron como las más nocivas en comparación a otras para el funcionamiento psicológico. Cuando este conflicto llevó al surgimiento de una enfermedad mental, la resistencia y las fragilidades de la personalidad hicieron que la descompensación pasara a su forma clínica definitiva. De este modo, la configuración sintomática (la actuación, la depresión, el bouffée délirante, las neurosis traumáticas, etc.) reflejaron más las características idiosincrásicas del paciente que la propia naturaleza de los 
problemas organizacionales causantes del desencadenamiento de la crisis psicopatológica.

3 Al contrario que la descompensación, la normalidad representó el resultado de un compromiso, de una lucha entre el sufrimiento causado por las demandas organizacionales y los mecanismos de defensa creados por los trabajadores en función de contener ese sufrimiento y evitar la descompensación. Sin embargo, estas estrategias de defensa, estrictamente ajustadas a las demandas de la organización del trabajo sobre el funcionamiento psicológico, tuvieron la marca específica y reconocible de la organización del trabajo en causa.

De esta forma, podemos describir las estrategias individuales y colectivas, cuya riqueza y diversidad constituyen la materia prima de un tipo de estudios clínicos llamados "la Clínica del Trabajo".

5 Pero el trabajo no es el único que generó sufrimiento psicológico o trastornos mentales. El mismo puede, dentro de determinadas formas de organización del trabajo, volverse un mediador importante de la génesis de placer en el trabajo y en la construcción del proceso de salud mental. Diciéndolo de otra manera, el trabajo nunca es neutro en relación a la salud mental. Él puede generar aquello que hay de peor (como el suicidio o los episodios clásicos de crisis) tanto como lo que hay de mejor : la realización personal a través del trabajo, la sublimación, el aporte a las obras de la cultura y de la civilización.

6 La tarea que le correspondió a la Clínica del Trabajo fue la de proveer un análisis de las condiciones que hacen oscilar la relación subjetiva con el trabajo hacia la patología o, al contrario, hacia la conquista de la identidad. Desde luego, la Psicopatología del Trabajo constituye apenas un capítulo específico dentro de la Clínica del Trabajo. Precisamente esta fue la razón que llevó al cambio de nombre de esta disciplina. El término "psicodinámica del trabajo" que se le dio a inicios de los años 90 contó con tres dimensiones :

- La primera dimensión implicó la expansión hacia el campo de la Clínica del Trabajo del que acabamos de hablar.

- La segunda dimensión se refirió a la existencia de una teoría y a una práctica específica. Esta teoría fue progresivamente elaborada a partir de 1980 gracias a un trabajo de investigación interdisciplinar desarrollado de forma ininterrumpida. Desde sus inicios, tres disciplinas fueron convocadas para este proceso :

- La Psicología : teniendo por elemento central la Teoría Psicoanalítica del sujeto, fundada en la metapsicología de S. Freud, y a la Teoría de la Seducción de J. Laplanche.

- La Sociología : teniendo por elemento central a la Sociología de la Ética, fundada sobre el abordaje comprensivo de W. Dilthey, M. Weber y A. Schütz.

- Las ciencias del trabajo: teniendo por elemento central a la ergonomía francófona de A. Ombredane, J.M. Faverge e A. Wisner.

- La tercera dimensión se relacionó con el método de investigación, cuyo objetivo resulta el análisis de la carga psíquica impuesta al sujeto debido al conflicto entre las demandas de la organización del trabajo y las propias demandas psicológicas, teniendo por objetivo el de evitar el riesgo de desestabilización de la identidad. El término "psicodinámica" fue un reflejo específico de la prioridad que se le dio, en este proceso de análisis, al conflicto psicológico a sus consecuencias.

7 Sin embargo, la psicodinámica del trabajo no representó a un tipo de investigación exclusivamente orientada hacia la producción de conocimientos científicos. Se 
encontró implicada de la misma forma con la acción, pues no hay investigación posible sin que exista un pedido emitido por un paciente, un trabajador, un colectivo de trabajo o una institución a través de su Departamento de Salud, Higiene y Seguridad del Trabajo; un servicio de salud del trabajo, un Sindicato, una Empresa, una Administración, etc. Esta acción, inseparable del proceso de producción del conocimiento, suscitó, a cambio, investigaciones teóricas y confrontaciones interdisciplinares en relación al tema de la teoría que dio origen a la acción.

De este modo, se desarrollaron nuevos conflictos interdisciplinares entre la teoría crítica y los filósofos de la Escuela de Frankfurt (A. Honneth, E. Renault, J.P. Deranty), con la Fenomenología, en particular, con la Fenomenología de la Vida (M. Henry).

Actualmente el debate continúa entre los investigadores provenientes de diversas disciplinas, no solamente entre la ergonomía y la medicina del trabajo, sino entre la psiquiatría, el psicoanálisis, la psicología, la psicología social, la antropología, la sociología, la historia, la lingüística, la economía, la tecnología, la ingeniería (Dejours, 1987).

Desde los años 70, cuando la psicodinámica del trabajo daba sus primeros pasos en el Laboratorio de Ergonomía de Alain Wisner, en el CNAM en París, la organización del trabajo atravesó varios momentos de mutación (la reestructuración de las tareas industriales, la aparición del modelo japonés, la aparición del modelo de gestión, las nuevas tecnologías y técnicas de información y de comunicación, el crecimiento de la economía en el sector del servicio, etc.). En este contexto, los trabajadores intentaron crear nuevos mecanismos de defensa para enfrentar al sufrimiento causado por las nuevas exigencias organizacionales, con más o menos éxito. Las patologías relacionadas con el trabajo aumentaron constantemente, al mismo tiempo que surgieron otras nuevas, lo que nos lleva a pensar que estos mecanismos de defensa creados no fueron suficientemente eficaces. A partir de la segunda mitad de los años 90 , las tentativas de suicidio y los suicidios se multiplicaron en los locales de trabajo, no sólo en Francia, sino también en Japón y China. Aunque hubo un aumento global de las riquezas en estos países, se observó un aumento de la violencia en el seno de estas sociedades, acompañado de una cronicidad creciente del fenómeno del desempleo y de un agravamiento de la pobreza. Paradójicamente, el aumento de la riqueza se correspondió con un aumento del sufrimiento humano y de las patologías. ¿Cómo explicar que tanto hombres como mujeres continuasen participando de una transformación del mundo y de la organización del trabajo que tendía a volverse contra ellos mismos, tal vez amenazando al "ser humano genérico" ? En este contexto, estábamos frente al regreso al tema de la alienación abordado en múltiples ocasiones desde finales del Siglo XVIII.

11 ¿Pudieron las investigaciones clínicas en el área de la psicodinámica del trabajo contribuir al análisis de este cambio significativo en el devenir de la "condición del hombre moderno" ? A finales de los años 90, la psicodinámica del trabajo inició un debate sobre el rol del trabajo en la génesis de nuevas formas de servidumbre voluntaria y sobre su responsabilidad en el deterioro de la salud mental durante el trabajo. Este análisis generó controversias con consecuencias que se manifiestan hasta hoy en el seno de las organizaciones sindicales y en el espacio público. Sin embargo, este debate no se restringió únicamente a Francia, donde los medios de comunicación, el cine, los documentales, el teatro; le confirieron una difusión particularmente importante durante los últimos años. En la actualidad, importantes estudios se realizan 
en varios países : en América del Norte, específicamente en Canadá; en América del Sur, específicamente en Brasil ; Europa, específicamente en Bélgica, Suiza y Alemania ; en Asia, específicamente en Japón y Taiwán ; y en Australia.

Pero, además de iniciar los debates entre disciplinas, la psicodinámica del trabajo también ocasionó controversias dentro de la propia área de la clínica. De hecho, en gran parte de los países y organizaciones internacionales, el estudio de las relaciones entre el trabajo y la salud mental fue esencialmente dominado por el tema del estrés y las investigaciones cuantitativas, estadísticas y epidemiológicas.

Más allá de las diferencias fundamentales que existieron en las bases científicas (referidas en la teoría del sujeto, la teoría social y la teoría del trabajo) opuestas a los modelos del estrés y de la psicodinámica del trabajo, debemos considerar las implicaciones prácticas de estas diferencias. Las acciones adoptadas como consecuencia de la teoría del estrés se tradujeron esencialmente en recomendaciones relacionadas con la gestión individual del estrés. De forma general, no criticaron a la organización del trabajo y se limitaron, por tanto, a un "tratamiento de los síntomas". Al contrario de este fenómeno, cualquier acción relacionada con la psicodinámica del trabajo buscó la identificación de los elementos para una intervención que no tuviese en cuenta sólo a los individuos, sino que integrase igualmente a la transformación de la organización del trabajo. Su objetivo fue, desde luego, la concepción de un "tratado etiológico" del sufrimiento y de las patologías mentales generadas por el trabajo.

Este tipo de orientación llevó a que los investigadores de la psicodinámica del trabajo llamasen la atención sobre el trabajo en colectivo y sobre el tema de las posibilidades de la cooperación. En efecto, las investigaciones en psicodinámica del trabajo durante los últimos diez años demostraron que la introducción y la rápida generalización de los nuevos métodos de evaluación individual del desempeño, llevados a cabo por las ciencias de la gestión, tuvieron un papel fundamental en la destrucción de las potencialidades del trabajo en colectivo, de cooperación y solidaridad. Así, los métodos de organización del trabajo estuvieron involucrados en los procesos de servidumbre voluntaria y de deterioro de la salud mental en el trabajo. En lugar de la ayuda mutua y la solidaridad, la soledad y el miedo invadieron el mundo del trabajo. De ahora en adelante, frente a la dominación, la injusticia, el asedio, la amenaza de despido ; cada trabajador estaba solo.

La glorificación del desempeño individual, desestructurando a las solidaridades, alcanzó a una de las garantías fundamentales para la salud mental en el trabajo. Para recuperar aquello que fue destruido en el transcurso de esta evolución nefasta, se hace necesaria una transformación de la organización del trabajo que permita restablecer las condiciones necesarias para la cooperación. De hecho, la psicodinámica del trabajo demostró que la cooperación no se puede separar de la actividad de producción de reglas laborales, que son al mismo tiempo reglas de savoir-vivre, de convivencia y de vida en conjunto.

16 El trabajo no sólo produce aquello que tenemos de mejor o de peor a nivel individual. Puede también generar aquello que hay de mejor a nivel colectivo, o sea, la armonía y el "vivre-ensemble" [1], así como lo peor, o sea, la soledad - y hasta la desolación -, la desconfianza, el miedo y la instrumentalización de los seres humanos, llegando al extremo de llevar a algunos de nosotros a quitar sus vidas frente a los propios colegas.

El tratamiento "etiológico" del sufrimiento del trabajo obliga a que nos apoyemos en una teoría y en una práctica para la reconstrucción del trabajo colectivo y del "vivre- 
ensemble". Esta teoría no podrá ser elaborada a partir de estudios cuantitativos sobre el estrés o la epidemiología.

Desde otra dimensión de la clínica, referida a los tratamientos de los enfermos que sufren patologías laborales, los principios del cuidar tendrán que ser diferentes si nos referimos a las teorías del estrés o a la psicodinámica del trabajo. Por tanto, es en ambas direcciones de acción (individual y colectiva) que la psicodinámica y las teorías del estrés toman caminos diferentes. Los artículos propuestos en el presente Volumen de la Revista Laboreal le darán al lector una idea del campo que se abre a la investigación científica y a la acción dentro del área de la clínica y la psicodinámica del trabajo.

\section{BIBLIOGRAFÍA}

Billiard, I. (2011). Santé mentale et travail : L'émergence de la psychopathologie du travail. La Dispute.

Dejours, C. (1987). Plaisir et souffrance dans le travail : Séminaire interdisciplinaire de psychopathologie du travail. Paris : Centre National de la Recherche Scientifique, 1987.

\section{NOTAS}

1. Nota del Traductor: literalmente "vivir en conjunto".

\section{AUTOR}

\section{CHRISTOPHE DEJOURS}

Conservatoire National des Arts et Métiers, 41 rue Gay-Lussac, 75005 Paris christophe.dejours@cnam.fr 